\title{
Vaccination antigrippale pour la saison 2019-2020 et risques relatifs à la COVID-19 : une étude cas/témoins au Québec
}

\author{
Jacques Pépin ${ }^{1 *}$, Philippe De Wals², Annie-Claude Labbé ${ }^{3,4}$, Alex Carignan', Marie-Elise Parent ${ }^{5}$, \\ Jennifer Yu ${ }^{5}$, Louis Valiquette ${ }^{1}$, Marie-Claude Rousseau ${ }^{5}$
}

\section{Résumé}

Contexte : Nous avons effectué une étude cas/témoins qui a examiné si l'administration du vaccin antigrippal inactivé au cours de la saison 2019-2020 a eu une incidence sur les risques relatifs à la maladie à coronavirus 2019 (COVID-19), car il y avait une préoccupation à savoir si le vaccin pourrait être préjudiciable par interférence virale.

Méthodes : Au total, 920 cas d'infection par le coronavirus du syndrome respiratoire aigu sévère 2 (SRAS-CoV-2) (diagnostiquée entre mars et octobre 2020) et 2123 témoins non infectés ont été recrutés parmi les personnes nées au Québec entre 1956 et 1976 et qui avaient reçu des services de diagnostic dans deux hôpitaux (Montréal et Sherbrooke, Québec). Après avoir obtenu le consentement, un questionnaire a été passé par téléphone. Les données ont été analysées par régression logistique.

Résultats : Chez les travailleurs de la santé, le vaccin antigrippal inactivé reçu au cours de la saison grippale précédente n'était pas associé à un risque accru de COVID-19 (rapport de cotes ajusté $[R C A]: 0,99$, intervalle de confiance $[I C]$ à $95 \%: 0,69-1,41)$. Parmi les participants qui n'étaient pas des travailleurs de la santé, la vaccination antigrippale était associée à des probabilités plus faibles de COVID-19 (RCA : 0,73, IC à $95 \% 0,56-0,96$ ).

Conclusion : Nous n'avons trouvé aucune preuve que le vaccin contre la grippe saisonnière augmentait le risque de développer la COVID-19.
Cette oeuvre est mise à la disposition selon les termes de la licence internationale Creative Commons Attribution 4.0

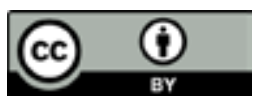

Affiliations

1 Université de Sherbrooke, Sherbrooke, QC

2 Université Laval, Québec, QC

${ }^{3}$ CIUSSS de l'Est-de-l'Ile-deMontréal, Montréal, QC

${ }^{4}$ Université de Montréal, Montréal, QC

${ }^{5}$ Institut national de la recherche scientifique, Laval, QC

*Correspondance :

jacques.pepin@usherbrooke.ca

Citation proposée : Pépin J, De Wals P, Labbé A-C, Carignan A, Parent M-E, Yu J, Valiquette L, Rousseau M-C. Vaccination antigrippale pour la saison 2019-2020 et risques relatifs à la COVID-19 : une étude cas/témoins au Québec. Relevé des maladies transmissibles au Canada 2021;47(10):477-82.

https://doi.org/10.14745/ccdr.v47i10a05f

Mots-clés : SRAS-CoV-2, COVID-19, grippe saisonnière, vaccin antigrippal

\section{Introduction}

Au début de la pandémie de maladie à coronavirus 2019 (COVID-19), certains ont émis I'hypothèse que le vaccin antigrippal inactivé pourrait paradoxalement accroître le risque de développer la COVID-19, et cette suggestion a été reprise par des mouvements anti-vaccins sur Internet. Une telle interférence virale a été décrite entre le vaccin antigrippal et les coronavirus (autres que le coronavirus du syndrome respiratoire aigu sévère 2; SRAS-CoV-2) bien que la validité de ces constatations ait été mise en doute $(1,2)$. Cette interférence a été signalée plus fréquemment chez les personnes qui avaient reçu le vaccin contre la grippe au cours de la saison 2017-2018. Une autre préoccupation a été qu'une surveillance sentinelle et trois autres études observationnelles ont montré que la réception du vaccin antigrippal trivalent pendant la saison 2008-2009 avait multiplié de 1,4 à 2,5 fois le risque d'infection avec le virus $\mathrm{H} 1 \mathrm{~N} 1$ pandémique et nécessitant un suivi médical au printemps-été 2009. Les auteurs ont proposé plusieurs mécanismes possibles pour leurs constatations (3).

L'objectif de la présente étude était de déterminer s'il y avait une interférence virale nuisible entre le vaccin antigrippal et I'infection par le SRAS-CoV-2 de sorte que la première augmente le risque de la seconde. Si tel était le cas, il faudrait en tenir compte dans la planification des prochaines campagnes de vaccination contre la grippe saisonnière. 


\section{Méthodologie}

Au milieu et à la fin de 2020, nous avons effectué une grande étude cas/témoins afin de déterminer si le vaccin BCG (bacille de Calmette-Guérin) (vaccin contre la tuberculose) administré pendant la petite enfance ou l'enfance, par son effet non spécifique sur l'immunité innée, offrait une protection à long terme contre l'infection par le SRAS-CoV-2 (les résultats de cette étude seront publiés ailleurs). Nous avons également inclus dans notre questionnaire une question exploratoire concernant la vaccination antigrippale au cours de la saison 2019-2020. Ces autodéclarations sont considérées comme fiables pour la saison la plus récente (4). Au total, 920 cas d'infection par le SRAS-CoV-2 confirmée par la réaction en chaîne de la polymérase (diagnostiquée entre mars et octobre 2020) et 2123 témoins non infectés (personnes qui $n$ 'ont jamais eu de test de réaction en chaîne de la polymérase du SRAS-CoV-2, positif ou négatif) ont été recrutés chez des personnes nées au Québec entre 1956 et 1976. L'identification des participants potentiels a été effectuée à l'aide des bases de données des laboratoires de microbiologie de l'Hôpital Maisonneuve-Rosemont à Montréal et du Centre Hospitalier Universitaire de Sherbrooke (CHUS). Les comités d'examen institutionnels de ces deux hôpitaux ont autorisé cette étude.

Pour les contrôles seulement, les critères d'exclusion ont été utilisés pour s'assurer qu'ils étaient relativement représentatifs de la population de l'ensemble des deux hôpitaux plutôt que de sa fraction la plus malade. Pour ce faire, nous avons exclu comme témoins potentiels les personnes qui ont été hospitalisées (pour une raison quelconque) ou qui sont allées aux urgences pendant la période d'étude, ainsi que celles qui fréquenté des cliniques où les patients immunodéprimés sont souvent reçus (hématologie, oncologie, rhumatologie, VIH, greffes rénales, dialyse, etc.). Les personnes vivant dans des établissements de soins de longue durée ont également été exclues en tant que cas ou contrôles, car la plupart n'auraient pu donner un consentement éclairé. Nous avons utilisé l'appariement fréquentiel sur le sexe et l'année de naissance, en visant deux contrôles par cas à l'Hôpital Maisonneuve-Rosemont et trois au CHUS.

Les personnes consentantes ont reçu par téléphone un questionnaire qui, après avoir vérifié leur admissibilité, a recueilli des données sociodémographiques et des renseignements sur la profession - travailleur de la santé (TdeS) ou non. Nous avons également vérifié le code postal à six caractères ensuite utilisé pour obtenir un indice de défavorisation matérielle fondé sur le recensement, conformément à une application élaborée par I'Institut national de santé publique du Québec (5). D'autres variables recueillies n'étaient pas liées à l'étude actuelle (e.g. cicatrice autodéclarée de BCG/variole, âge lors du BCG, etc.).

Des analyses univariées et multivariées ont été effectuées par régression logistique inconditionnelle, en utilisant la version R 4.0.2 (6). Les facteurs de confusion possibles, qui auraient pu être liés à la vaccination contre le SRAS-CoV-2 et la grippe, comprenaient l'âge (en tant que variable continue), le sexe, I'hôpital de recrutement, le quintile de défavorisation matérielle fondé sur le recensement et le statut de travailleur de la santé. Nous avons choisi d'ajuster pour tous ces facteurs de confusion a priori, indépendamment de leur contribution au modèle. On a évalué la modification des effets selon le statut TdeS, le sexe et l'âge en incluant un terme d'interaction dans trois modèles de régression distincts, comprenant tous les facteurs de confusion possibles (statut du TdeS*vaccin antigrippal, sexe*vaccin antigrippal, groupe d'âge*vaccin antigrippal) afin d'obtenir une valeur $p$ pour chaque terme $d$ 'interaction. Des analyses stratifiées selon le statut TdeS, le sexe et le groupe d'âge ont également été effectuées pour estimer les rapports de cotes et les intervalles de confiance de $95 \%$ pour l'association entre la vaccination antigrippale et le SRAS-CoV-2 dans ces sousgroupes.

Il manquait des données sur la vaccination antigrippale pour 42 cas et 16 témoins. L'échantillon analytique comprenait donc 878 cas et 2107 témoins pour lesquels cette information était disponible.

Il manquait des données pour l'indice de défavorisation matérielle (indisponibles pour les récents développements résidentiels et les codes postaux où plus de $15 \%$ de la population vivait dans un établissement) pour $6,3 \%$ des participants (56 cas et 132 témoins). Afin de régler ce problème et éviter d'exclure les sujets ayant un statut de vaccination antigrippale connue, on a effectué une imputation multiple par équations chaînées pour cette variable (20 ensembles de données imputées).

\section{Résultats}

Les caractéristiques des cas et des témoins sont présentées au tableau 1. Comme on pouvait s'y attendre, étant donné que l'étude a été menée avant la disponibilité des vaccins contre le SRAS-CoV-2, il y avait plus de TdeS dans les cas que dans les témoins.

\section{Tableau 1 : Caractéristiques des cas et des témoins}

\begin{tabular}{|c|c|c|c|c|}
\hline \multirow{2}{*}{ Caractéristiques } & \multicolumn{2}{|c|}{$\begin{array}{c}\text { Cas } \\
\mathbf{n}=\mathbf{8 7 8}\end{array}$} & \multicolumn{2}{|c|}{$\begin{array}{c}\text { Témoins } \\
\mathbf{n}=\mathbf{2} 107\end{array}$} \\
\hline & $n$ & $\%$ & $\mathbf{n}$ & $\%$ \\
\hline \multicolumn{5}{|l|}{ Sexe } \\
\hline Hommes & 333 & 37,9 & 814 & 38,6 \\
\hline Femmes & 545 & 62,1 & 1293 & 61,4 \\
\hline \multicolumn{5}{|l|}{ Âge (années) } \\
\hline 44 à 49 & 213 & 24,3 & 525 & 24,9 \\
\hline 50 à 54 & 213 & 24,3 & 465 & 22,1 \\
\hline 55 à 59 & 250 & 28,5 & 579 & 27,5 \\
\hline 60 à 64 & 202 & 23,0 & 538 & 25,5 \\
\hline
\end{tabular}


Tableau 1 : Caractéristiques des cas et des témoins (suite)

\begin{tabular}{|c|c|c|c|c|}
\hline \multirow{2}{*}{ Caractéristiques } & \multicolumn{2}{|c|}{$\begin{array}{c}\text { Cas } \\
\mathbf{n}=\mathbf{8 7 8}\end{array}$} & \multicolumn{2}{|c|}{$\begin{array}{c}\text { Témoins } \\
n=2107\end{array}$} \\
\hline & $\mathbf{n}$ & $\%$ & $\mathbf{n}$ & $\%$ \\
\hline \multicolumn{5}{|l|}{ Hôpital } \\
\hline $\begin{array}{l}\text { Maisonneuve- } \\
\text { Rosemont }\end{array}$ & 591 & 67,3 & 1226 & 58,2 \\
\hline CHUS & 287 & 32,7 & 881 & 41,8 \\
\hline \multicolumn{5}{|c|}{ Défavorisation matérielle } \\
\hline Inférieur & 149 & 17,0 & 292 & 13,9 \\
\hline Bas & 159 & 18,1 & 386 & 18,3 \\
\hline Moyenne & 163 & 18,6 & 442 & 21,0 \\
\hline Haut & 202 & 23,0 & 460 & 21,8 \\
\hline Plus élevé & 149 & 17,0 & 395 & 18,7 \\
\hline Manquant & 56 & 6,4 & 132 & 6,3 \\
\hline \multicolumn{5}{|l|}{ Travail } \\
\hline $\begin{array}{l}\text { Milieux de soins de } \\
\text { santé }\end{array}$ & 425 & 48,4 & 231 & 11,0 \\
\hline Tous les autres & 453 & 51,6 & 1876 & 89,0 \\
\hline
\end{tabular}

Tableau 2 : Vaccin contre la grippe au cours de la saison 2019-2020 parmi les cas de COVID-19 et les témoins non infectés

\begin{tabular}{|c|c|c|c|c|c|c|c|c|c|}
\hline \multirow{2}{*}{ Caractéristiques } & \multicolumn{2}{|c|}{$\begin{array}{c}\text { Cas } \\
\mathrm{n}=878\end{array}$} & \multicolumn{2}{|c|}{$\begin{array}{c}\text { Témoins } \\
n=2107\end{array}$} & \multicolumn{2}{|c|}{ Brut } & \multicolumn{2}{|c|}{ Ajusté } & \multirow{2}{*}{$\begin{array}{l}\text { valeur } p \text { pour } \\
\text { l'interaction }^{a}\end{array}$} \\
\hline & $\mathbf{N}$ & $\%$ & $\mathbf{N}$ & $\%$ & $\begin{array}{l}\text { Rapport } \\
\text { des cotes }\end{array}$ & IC à $95 \%$ & $\begin{array}{l}\text { Rapport } \\
\text { des cotes }\end{array}$ & IC à $95 \%$ & \\
\hline \multicolumn{10}{|l|}{ Tous les participants } \\
\hline Non vacciné & 649 & 73,9 & 1626 & 77,2 & 1,00 & s.o & 1,00 & s.o. & \multirow{2}{*}{ s.o. } \\
\hline Vacciné & 229 & 26,1 & 481 & 22,8 & 1,19 & $0,99-1,43$ & 0,81 & $0,66-1,00^{b}$ & \\
\hline \multicolumn{10}{|l|}{ Travailleur de la santé } \\
\hline Non vacciné & 273 & 64,2 & 149 & 64,5 & 1,00 & s.o. & 1,00 & s.o. & \multirow{2}{*}{0,14} \\
\hline Vacciné & 152 & 35,8 & 82 & 35,5 & 1,01 & $0,72-1,42$ & 0,99 & $0,69-1,41^{c}$ & \\
\hline \multicolumn{10}{|l|}{ Pas travailleur de la santé } \\
\hline Non vacciné & 376 & 83,0 & 1477 & 78,7 & 1,00 & s.o. & 1,00 & s.o. & \multirow{2}{*}{0,14} \\
\hline Vacciné & 77 & 17,0 & 399 & 21,3 & $0,76^{c}$ & $0,58-0,99^{c}$ & 0,73 & $0,56-0,96^{\mathrm{c}, \mathrm{d}}$ & \\
\hline \multicolumn{10}{|l|}{ Hommes } \\
\hline Non vacciné & 252 & 75,7 & 645 & 79,2 & 1,00 & s.o. & 1,00 & s.o. & \multirow{2}{*}{0,73} \\
\hline Vacciné & 81 & 24,3 & 169 & 20,8 & 1,23 & $0,90-1,66$ & 0,87 & $0,62-1,23^{e}$ & \\
\hline \multicolumn{10}{|l|}{ Femmes } \\
\hline Non vacciné & 397 & 72,8 & 981 & 75,9 & 1,00 & s.o. & 1,00 & s.o. & \multirow{2}{*}{0,73} \\
\hline Vacciné & 148 & 27,2 & 312 & 24,1 & 1,17 & $0,93-1,47$ & 0,78 & $0,60-1,01^{\mathrm{e}}$ & \\
\hline \multicolumn{10}{|l|}{ Entre 44 et 54 ans } \\
\hline Non vacciné & 321 & 75,4 & 812 & 82,0 & 1,00 & s.o. & 1,00 & s.o. & \multirow{2}{*}{0,86} \\
\hline Vacciné & 105 & 24,6 & 178 & 18,0 & $1,49^{c}$ & $1,13-1,96^{c}$ & 0,85 & $0,62-1,17^{f}$ & \\
\hline \multicolumn{10}{|l|}{ Entre 55 et 64 ans } \\
\hline Non vacciné & 328 & 72,6 & 814 & 72,9 & 1,00 & s.o. & 1,00 & s.o. & \multirow{2}{*}{0,86} \\
\hline Vacciné & 124 & 27,4 & 303 & 27,1 & 1,02 & $0,79-1,30$ & 0,82 & $0,62-1,08^{f}$ & \\
\hline
\end{tabular}

Un tiers des travailleurs de la santé et un cinquième des autres travailleurs avaient été vaccinés contre la grippe. Les résultats de la régression logistique univariée et multivariée sont présentés au tableau 2. Le vaccin antigrippal inactivé au cours de la saison 2019-2020 n'était pas associé à la COVID-19 chez les TdeS. Parmi les participants qui n'étaient pas des travailleurs de la santé, il était associé à des probabilités plus faibles de COVID-19. Cependant, il n'y avait aucune indication d'interaction au moment d'examiner le terme d'interaction. L'association entre la vaccination antigrippale et la COVID-19 ne différait pas selon le sexe ou le groupe d'âge en fonction des estimations de l'association ou des valeurs $p$ ou des termes d'interaction (tableau 2).

\section{Discussion}

Nous avons constaté que parmi les participants qui ne sont pas des TdeS, le vaccin contre la grippe saisonnière était associé à des probabilités plus faibles d'infection par le SRAS-CoV-2 et non à un risque accru, comme nous l'avions initialement envisagé. Aucun effet du vaccin contre la grippe saisonnière sur les probabilités d'infection par le CoV-2 du SRAS n'a été 
observé chez les TdeS. II n'y a aucune raison de croire que le vaccin antigrippal pourrait offrir une protection croisée contre le SRAS-CoV-2 au moyen de mécanismes immunitaires adaptatifs, étant donné la dissimilarité des protéines superficielles de ces deux virus. Une hypothèse possible pour expliquer cet effet protecteur apparent chez ceux qui ne sont pas des TdeS est que la protection dérivée du vaccin contre la grippe au printemps 2020 (son efficacité au Canada a été estimée à $58 \%$ ) (7) pourrait avoir réduit les chances de consulter pour des symptômes d'infection des voies respiratoires supérieures liés à la grippe permettant à une infection concomitante au SRAS-CoV-2 d'être diagnostiquée, ou pourrait avoir réduit le risque d'un SRAS plus grave (donc mieux détecté) en présence $d^{\prime}$ 'une double infection. De telles co-infections sont cependant assez rares. Au Royaume-Uni, au cours de la première vague de COVID-19 (janvier-avril 2020), sur 19256 personnes testées, seulement 58 avaient une double infection, alors que 992 n'avaient qu'une grippe et 4442 n'avaient qu'une infection par le SRAS-CoV-2 (8). Des résultats semblables ont été rapportés en Californie (9). De plus, au Canada, la circulation du virus de la grippe a pris fin en mars 2020, et la grande majorité de nos cas COVID-19 ont été signalés après cette date (10).

Il est plus plausible que les personnes qui ne sont pas des TdeS et qui reçoivent le vaccin antigrippal saisonnier, dont certaines ont des maladies chroniques, se préoccupent davantage de leur santé en général, de sorte qu'elles ont peut-être été plus conformes à la distanciation physique et à l'utilisation de masques, ou qu'elles aient réduit leur exposition potentielle en restant à la maison. Ces mesures de santé publique auraient réduit leur risque d'infection par le SRAS-CoV-2; une variante du phénomène connu sous le nom de biais du vacciné sain (11). Cela n'a peut-être pas été le cas des TdeS qui se savaient être à risque élevé de contracter la COVID-19 professionnelle, et qui, par conséquent, auraient été très prudents de façon consistante afin de réduire leur exposition au SRAS-CoV-2.

Dans un examen systématique datant d'octobre 2020, Del Riccio a identifié sept études méthodologiquement solides qui avaient examiné cette association, et les personnes vaccinées contre la grippe étaient moins susceptibles d'avoir la COVID-19 dans cinq de ces études (12). Des publications plus récentes ont également montré que le vaccin antigrippal était associé à des probabilités plus faibles d'infection par le SRAS-CoV-2 aux États-Unis (13-15) et en Israël (16), alors qu'une étude américaine plus petite n'a pas documenté les effets (17). La plus grande étude, qui compte 137037 personnes de la base de données électronique sur les dossiers de santé de la Mayo Clinic, a montré une faible probabilité de développer la COVID-19 non seulement chez les personnes vaccinées contre la grippe, mais aussi chez celles qui ont reçu des vaccins conjugués contre la polio, l'Haemophilus influenzae de type $B$, la rougeole, les oreillons et la rubéole, la varicelle, I'hépatite $B$, I'hépatite $A$ ou le pneumocoque (15). De telles associations avec des produits vaccinaux multiples et non apparentés suggèrent un effet « d'utilisateur sain » ou « de vacciné sain ».
Une limite de l'étude était que nous n'avons pas recueilli de données sur les comorbidités puisque cela ne pouvait pas confondre l'association entre le BCG et la COVID-19, l'objectif principal de cette étude (cela aurait exigé que ces maladies soient associées à l'administration du BCG quatre à six décennies plus tôt, un scénario très improbable). Cependant, parmi les participants qui n'étaient pas TdeS, les indications pour le vaccin antigrippal comprennent certaines maladies (diabète, obésité, maladies cardiaques ou pulmonaires, etc.) qui sont elles-mêmes associées à des formes graves de la COVID-19, et donc plus sujettes à être testées. Un ajustement pour ces facteurs de confusion potentiels aurait pu modifier légèrement la mesure de I'association entre le vaccin contre la grippe et la COVID-19 vers la valeur nulle si l'atténuation des risques chez les vaccinés était plus marquée chez les patients atteints de comorbidité.

Une autre limite de notre étude est que nous avons étudié des personnes âgées de 44 à 64 ans, alors que la principale cible de la vaccination contre la grippe saisonnière est le groupe âgé de 65 ans ou plus. II semble toutefois peu probable qu'une interférence virale entre le SRAS-CoV-2 et le vaccin antigrippal saisonnier varie en fonction de l'âge.

Enfin, comparativement aux témoins, une proportion beaucoup plus élevée de cas (48\%) étaient des TdeS. Cela reflétait le portrait épidémiologique global de la COVID-19 au Québec au cours de la première vague, lorsque les TdeS étaient très à risque d'infection professionnelle et représentaient $41 \%$ des cas chez les personnes de 18 à 59 ans (18). Dans ce contexte, un biais de sélection semble peu probable, mais nous ne pouvons exclure la possibilité que les TdeS aient différé des autres participants dans leur souvenir de la vaccination antigrippale au cours de la saison précédente en raison d'un biais de désirabilité sociale. Toutefois, un tel biais semble peu probable étant donné que seulement $36 \%$ des TdeS ont rapporté avoir été vaccinés, ce qui est comparable aux données de surveillance courante de la vaccination antigrippale dans les établissements de soins de santé du Québec.

\section{Conclusion}

Nous n'avons trouvé aucune preuve que le vaccin antigrippal saisonnier augmentait le risque de développer la COVID-19 et la stratégie de vaccination habituelle n'a pas besoin d'être modifiée pour la saison 2021-2022.

\section{Déclaration des auteurs}

A. C. L., J. P., P. D. W., M. C. R., M. E. P. - A conçu l'étude, analysé et interprété les données, rédigé et édité le manuscrit M. C. R., J. Y. - Analyse des données

A. C., L. V. - A contribué à l'interprétation des données et à la rédaction du manuscrit

Tous les auteurs ont approuvé la version finale du manuscrit. 
Le contenu et les opinions exprimées dans cet article sont ceux des auteurs et ne correspondent pas nécessairement ceux du gouvernement du Canada.

\section{Intérêts concurrents}

Aucun.

\section{Remerciements}

Les personnes suivantes ont contribué à la collecte de données (par ordre alphabétique) : K. A. Baki, D. Ag Bazet, M.-A. Binette, J. Boisvert, M.-P. Boisvert, J. Bourget, V. Choinière, A. Delimi, A. Deneault, V. Dumont, L. Duquette-Laplante, R. Escobar Careaga, K. Farag, L. Foudil, S. Gélin, K. Gendron, L.-A. Gervais, O. Grimard, R. Harti, R. Lachance, A. Marcil-Héguy, N. Métayer, S. Payeur, J.-C. Pellerin, C. Simard, R. Thibeault, A.-S. Thiffault et K. Vettese. Nous sommes également reconnaissants à M. Malachy, J.-H. Lee et N. Frappier pour leur aide dans les bases de données des hôpitaux, à N. Gagnon pour son aide dans la mise en place de l'interface de saisie des données et à $G$. Deceuninck pour ses suggestions utiles.

\section{Financement}

Ce travail a été soutenu par le Centre de recherche du centre hospitalier universitaire de Sherbrooke grâce à un financement d'urgence spécial COVID-19 fourni par la Fondation du centre hospitalier universitaire de Sherbrooke. Le bailleur de fonds n'a joué aucun rôle dans la conception des études, dans la collecte, l'analyse et l'interprétation des données, dans la rédaction du rapport ni dans la décision de soumission.

\section{Références}

1. Wolff GG. Influenza vaccination and respiratory virus interference among Department of Defense personnel during the 2017-2018 influenza season. Vaccine 2020;38(2):350-4. DOI PubMed

2. Skowronski DM, Zou M, Clarke Q, Chambers C Dickinson JA, Sabaiduc S, Olsha R, Gubbay JB, Drews SJ, Charest $H$, Winter $A L$, Jassem A, Murti M, Krajden $M$, De Serres $G$. Influenza vaccine does not increase the risk of coronavirus or other noninfluenza respiratory viruses: retrospective analysis from Canada, 2010-11 to 2016-17. Clin Infect Dis 2020;71(16):2285-8. DOI PubMed
3. Skowronski DM, De Serres G, Crowcroft NS, Janjua NZ, Boulianne N, Hottes TS, Rosella LC, Dickinson JA, Gilca R, Sethi P, Ouhoummane N, Willison DJ, Rouleau I, Petric M, Fonseca K, Drews SJ, Rebbapragada A, Charest H, Hamelin ME, Boivin G, Gardy JL, Li Y, Kwindt TL, Patrick DM, Brunham RC; Canadian SAVOIR Team. Association between the 2008-09 seasonal influenza vaccine and pandemic H1N1 illness during Spring-Summer 2009: four observational studies from Canada. PLoS Med 2010;7(4):e1000258. DOl PubMed

4. King JP, McLean HQ, Belongia EA. Validation of self-reported influenza vaccination in the current and prior season. Influenza Other Respir Viruses 2018;12(6):808-13. DOI PubMed

5. Institut National de Santé Publique du Québec. Indice de défavorisation matérielle et sociale (accédé 2021-07-21). https://www.inspq.qc.ca/defavorisation/indice-dedefavorisation-materielle-et-sociale

6. The R Foundation. The R Project for Statistical Computing. Vienna (Austria): R Foundation; 2020.

https://www.R-project.org/

7. Skowronski DM, Zou M, Sabaiduc S, Murti M, Olsha R, Dickinson JA, Gubbay JB, Croxen MA, Charest H, Jassem A, Krajden M, Bastien N, Li Y, De Serres G. Interim estimates of 2019/20 vaccine effectiveness during early-season co-circulation of influenza A and B viruses, Canada, February 2020. Euro Surveill 2020;25(7):2000103. DOI PubMed

8. Stowe J, Tessier E, Zhao H, Guy R, Mulller-Pebody B, Zambon M, Andrews N, Ramsay M, Lopez Bernal J. Interactions between SARS-CoV-2 and influenza and the impact of coinfection on disease severity: a test negative design. Int J Epidemiol 2021;dya081. DOI

9. Kim D, Quinn J, Pinsky B, Shah NH, Brown I. Rates of co-infection between SARS-CoV-2 and other respiratory pathogens. JAMA 2020;323(20):2085-6. DOI PubMed

10. Agence de la santé publique du Canada. Surveillance de l'influenza : Du 5 avril au 9 mai 2020 (semaines de déclaration 15 à 19). https://www.canada.ca/fr/santepublique/services/publications/maladies-et-affections/ surveillance-influenza/2019-2020/semaines-15-19-5-avril-9mai-2020.html

11. Remschmidt C, Wichmann $\mathrm{O}$, Harder T. Frequency and impact of confounding by indication and healthy vaccinee bias in observational studies assessing influenza vaccine effectiveness: a systematic review. BMC Infect Dis 2015;15:429. DOI PubMed

12. Del Riccio M, Lorini C, Bonaccorsi G, Paget J, Caini S. The association between influenza vaccination and the risk of SARS-CoV-2 infection, severe illness, and death: a systematic review of the literature. Int J Environ Res Public Health 2020;17(21):7870. DOI PubMed 
13. Huang K, Lin SW, Sheng WH, Wang CC. Influenza vaccination and the risk of COVID-19 infection and severe illness in older adults in the United States. Sci Rep 2021;11(1):11025. DOI PubMed

14. Zein JG, Whelan G, Erzurum SC. Safety of influenza vaccine during COVID-19. J Clin Transl Sci 2020;5 e49:1-3. DOI

15. Pawlowski $C$, Puranik $A$, Bandi $H$, Venkatakrishnan AJ, Agarwal V, Kennedy R, O'Horo JC, Gores GJ, Williams AW, Halamka J, Badley AD, Soundararajan V. Exploratory analysis of immunization records highlights decreased SARS-CoV-2 rates in individuals with recent non-COVID-19 vaccinations. Sci Rep 2021;11(1):4741. DOI PubMed
16. Green I, Ashkenazi S, Merzon E, Vinker S, Golan-Cohen A. The association of previous influenza vaccination and coronavirus disease-2019. Hum Vaccin Immunother 2021;17(7):2169-75. DOl PubMed

17. King JP, McLean HQ, Belongia EA. Risk of symptomatic severe acute respiratory syndrome coronavirus 2 infection not associated with influenza vaccination in the 2019-2020 season. Influenza Other Respir Viruses 2021. DOI PubMed

18. Institut National d'Excellence en Santé et Services Sociaux. Première vague de la pandémie de COVID-19 au Québec : regard sur les facteurs associés aux hospitalisations et aux décès. https://www.inesss.qc.ca/fileadmin/doc/INESSS/ COVID-19/COVID-19_INESSS_EP_Portrait_cohorte_COVID. pdf

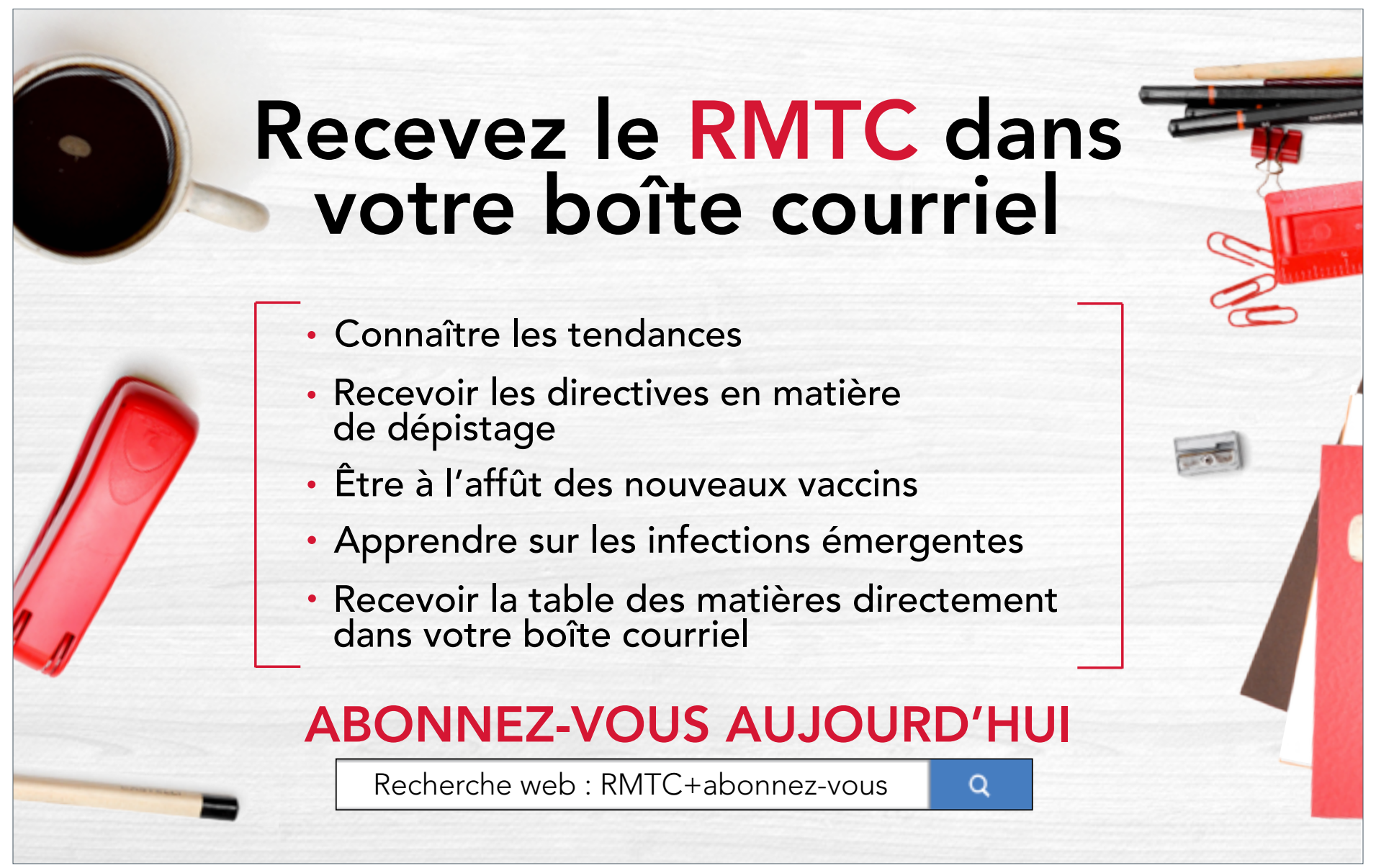

